\title{
Shared Path Protection for Distance Adaptive Elastic Optical Networks under Dynamic Traffic
}

(Invited Paper)

\author{
Aras Tarhan \\ Communication Systems, School of ICT \\ KTH Royal Institute of Technology \\ Kista, Sweden \\ atarhan@kth.se
}

\author{
Cicek Cavdar \\ Communication Systems, School of ICT \\ KTH Royal Institute of Technology \\ Kista, Sweden \\ cavdar@kth.se
}

\begin{abstract}
Elastic Optical Networks (EONs), evolved as a scalable infrastructure to provide optical connectivity for large variety of bandwidth requests ranging from 1 Gbps to 1 Tbps. Thanks to the enabling technologies such as bandwidth variable transponders and flexible switches, bandwidth adaptive spectrum allocation became possible. EONs can carry large optical channels with higher spectrum efficiency with the recent changes in the standard fixed division of optical spectrum. In this study we propose a distance adaptive, dynamic shared path protection scheme for EONs. In conventional WDM networks, shareability used to be one of the prime objectives to maximize the backup resource efficiency. In EONs, spectrum resources can be shared partially between connections and different parts of the allocated spectrum may be shared by different connections at the same time. Not only the routing but also spectrum allocation of backup resources has a big impact on the shareability in EONs. Taking this into account, we developed a novel RSA (Routing and Spectrum Allocation) algorithm applying different strategies for primary and backup resources called Primary First-Fit Modified Backup Last-Fit (PF-MBL) aiming to reduce the fragmentation and to increase the shareability. As a result overall bandwidth blocking probability is significantly reduced in the network. Results show that PF-MBL can improve the performance in terms of bandwidth blocking probability by $24 \%$ up to $59 \%$ compared to the current outperforming algorithm when the bandwidth acceptance ratio of the system varies from $90 \%$ to $99.9 \%$ in different loads.
\end{abstract}

Keywords- Elastic Optical Networks; Flexible Spectrum; Shared Path Protection; Survivability.

\section{INTRODUCTION}

Recently, the Internet traffic demand has been rising up by approximately $40 \%$ every year, corresponding to the doubling of the demand every two years [1]. Furthermore, it is very likely that this trend will continue due to the massively increasing use of Internet services such as Video on Demand (VoD), high definition Internet Protocol (IP) TV, cloud computing and grid applications requiring high amount of data rate.

Wavelength Division Multiplexing (WDM) technique has mainly been used in optical backbone networks. To meet these high data rate demands, modulation formats installing higher number of bits per symbol into $50 \mathrm{GHz}$ fixed grid spaces along the frequency spectrum were brought in. For instance, 100
$\mathrm{Gb} / \mathrm{s}$-based transmission systems have been fit into $50 \mathrm{GHz}$ fixed grid space and commercialized in the last three years [2]. However, it is seen infeasible to fit data rates over $100 \mathrm{~Gb} / \mathrm{s}$ such as $400 \mathrm{~Gb} / \mathrm{s}$ and $1 \mathrm{~Tb} / \mathrm{s}$ into this fixed grid space [2]. Since wavelength-routed networks require full allocation of a wavelength to a connection even if the whole grid is not necessary, a wide range of data rate in traffic demands leads to some residual frequency parts wasted in the fixed grids. Elastic optical networks (EONs) evolved as a response to these drawbacks of fixed grid wavelength routed networks. Efficient spectrum allocation is achieved thanks to the flexible grid and provisioning end-to-end optical elastic channels become possible to carry variable traffic demands.

Survivability plays an important role in EONs. A link failure may lead to a huge data loss for data rates between $1 \mathrm{~Gb} / \mathrm{s}$ and $1 \mathrm{~Tb} / \mathrm{s}$, therefore resource efficient protection techniques need to be developed to guarantee the recovery of such failures. Shared path protection, studied significantly in WDM optical networks has a big potential to solve this problem in EONs if backup resource sharing is done carefully and simple techniques are developed to reduce fragmentation problem which leads to high blocking rates due to unavailability of link disjoint primary and backup path pairs.

In this study we develop a novel algorithm for the problem of shared path protected routing, modulation format and spectrum assignment (SPP-RMLSA) under dynamic traffic conditions. We mainly focus on the RMLSA of backup resources and the impact on the overall fragmentation to reduce the bandwidth blocking probability (BBP) of the network. Our algorithm aims to increase the number of shared frequency slots among backup paths as well as to decrease the fragmentation of spectrum resources. We compare our solution with two existing solutions, [3] and [4], used for resource allocation by simulation.

The rest of the paper is organized as follows. In sub-section 2 , we present the problem statement and the constraints. Subsection 3 describes briefly existing solutions and presents a novel algorithm for SPP-RMLSA under dynamic traffic conditions. The performance is evaluated compared to the existing approaches by software-based simulations in section 4 together with the results. Finally, we present the conclusion. 


\section{Shared Path Protection In Elastic Optical NETWOKS}

Under dynamic traffic scenario, shared path protected (SPP) routing, modulation format and spectrum assignment (RMLSA) problem in elastic optical networks can be stated as follows.

\section{A. Given}

a) Physical topology of a network represented by $G(N, L)$ where $N$ is the set of nodes and $L$ is the set of connecting bidirectional optical links; b) the number of frequency slots on each link represented by $W$; c) $D_{i j}$ denoting the distance of link $i-j \in \mathrm{L} ; \mathrm{d})$ a connection request $R=\left\{s, d, b, t_{a}, t_{h}\right\}$ between a source-destination pair $(s, d)$ with bitrate $b$ (in $b p s$ ), arrival time $t_{a}$ and holding time $t_{h} ;$ e) current availability of a frequency slot $k$ in link $i-j$ represented by a binary $S_{k} ; \mathrm{f}$ ) set of modulation levels $M=\{m, c, t\}$ where $m$ is the modulation format, $c$ is the subcarrier capacity and $t$ is the transmission reach of the corresponding modulation format.

\section{B. Output}

A shared-path-protected connection $C$ as shown below where $l_{w}$ and $l_{b}$ denote working and backup paths, $M$ denotes the modulation format, $f_{a}$ and $f_{z}$ are the starting and ending frequencies of the contiguous spectrum allocated for the demand, and $t_{e}$ denotes expiry time: $C=\left\{l_{w}, l_{b}, M, f_{a}, f_{z}, t_{e}\right\}$.

\section{Objective}

Minimize connection blocking probability and bandwidth blocking probability.

\section{Constraints}

The objective is subject to the following constraints.

- Spectrum continuity: This constraint requires that, for a given connection request, the same segment of the spectrum needs to be allocated in every link along the route, i.e., the same set of frequency slot numbers must be assigned.

- Subcarrier consecutiveness: Frequency slots that are assigned to the same connection request must be adjacent to each other in one link.

- Frequency slot capacity: Each link has a limited number of frequency slots to be allocated. This number is the same in all links.

- Non-overlapping spectrum assignment: Allocated spectrum slices for each connection request must be separated by guard bands in order to prevent interference.

- Link-disjointness for primary and backup paths of a connection: The primary and the backup path of a given connection must be link disjoint so as to provide two independent routes for a connection.

- Backup path sharing: Connections belonging to a common set of shared risk link group (SRLG) cannot share backup resources. SRLG is defined as the set of connections, primary paths of which are sharing a link.

- Transmission distance: In transparent optical networks, one bit increase in the modulation format of the transmission, requires to halve the transmission distance in order to keep the same level of Quality of transmission (QoT) [5], as shown in Table 1. Hence, modulation format should be lowered as the transmission distance of the optical path increases in order to resist against deterioration.

TABLE I. SUB-CARRIER CAPACITY AND OPTICAL REACH FOR DIFFERENT MODULATION FOTMATS [5]

\begin{tabular}{rrr}
\hline $\begin{array}{r}\text { Modulation Format } \\
\text { BPSK }\end{array}$ & $\begin{array}{r}\text { Subcarrier Capacity } \\
\text { (Gbit/sec) }\end{array}$ & Transparent Reach (km) \\
QPSK & 12.5 & 4000 \\
8-QAM & 25 & 2000 \\
16-QAM & 37.5 & 1000 \\
32-QAM & 50 & 500 \\
64-QAM & 62.5 & 250 \\
\hline
\end{tabular}

\section{Shared PATH Protected RMLSA}

It is proved that RMLSA problem is NP-complete by drawing an analogy between RMLSA problem and a well-known NP-complete problem, Multiprocessor Scheduling [6]. As a result, Integer Linear Programming (ILP) cannot scale for large data sets. Therefore, we have focused on heuristic algorithms to solve the RMLSA problem.

Examined algorithms and the proposed algorithm employ two-step approach in order to decrease the complexity. In twostep approach, RMLSA problem is split into two sub-problems, routing and spectrum assignment respectively. Then, they are solved sequentially. For routing, k-shortest paths are calculated for a given source and destination pair. Later, these paths are examined for available spectrum segments in spectrum assignment part. One path, if possible, is selected among those, which satisfy spectrum assignment constraints, with respect to the given heuristic algorithm. In order to find k-shortest paths, Yen's Algorithm has been used [7]. Application of this model for the dynamic traffic will be as follows: k-shortest paths are found for the primary path when a connection request arrives in the network. Then, one path out of the candidate paths that satisfy spectrum assignment constraints is chosen. Following the primary path's RMLSA, the same procedure will be applied for finding the backup path ensuring link-disjointness constraint. In contrast to primary path spectrum assignment, frequency slots employed by backup resources are treated as 'nonutilized' except from the ones whose primary paths have common link(s) with the current primary path violating the backup path sharing constraint. In this way, backup resources are shared among backup paths while ensuring survivability against single-link failure.

The number of frequency slots to be assigned is determined with respect to the length of the path selected since modulation format changes depending on the transmission distance. In case there is no solution exists in search of either of primary or backup, the connection request is blocked. 


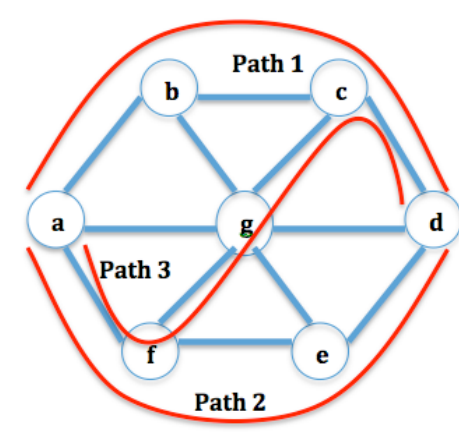

(a) k-shortest backup path candidates from node a to node $\mathrm{d}$.

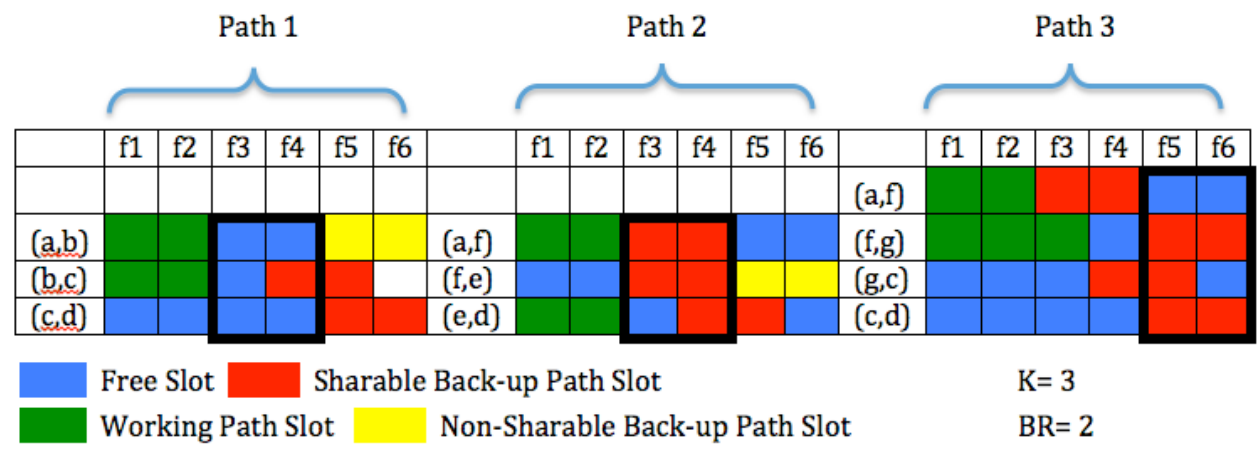

(b) Backup spectrum assignments provided that BR (bitrate) requires 2 frequency slots in every path ${ }^{1}$

Figure 1. Illustration of backup spectrum assignments in different heuristics where BR denotes the required number of frequency slots and $\mathrm{K}$ denotes the number of k-shortest paths

Three spectrum assignment algorithms combined with kshortest path will be described: First-Fit (FF) Algorithm proposed in [3], Minimum Free Spectrum Block Consumption Algorithm (MFSB) proposed in [4] $]^{1}$ and a novel solution Primary First-Fit Modified Backup Last-Fit (PF-MLF) proposed for the first time in this paper.

Fig. 1 illustrates backup path spectrum assignment. In this example, connection requests require equal bit rates corresponding to two slots. For the sake of clarity we skip the modulation format assignment step, $\mathrm{k}$ is equal to three in $\mathrm{k}$-shortest path and there are six frequency slots in each link. Fig 1 (a) shows the three shortest paths for backup path spectrum assignment where Path 1 is the shortest and Path 3 is assumed to be the longest. Green color in a frequency slot represents that it is being utilized by a primary path. Blue color signifies that a slot is idle meaning that it is utilized neither by a primary path nor by backup paths and free to be reserved. Red colored frequency slots are already reserved by other connections and are also able to be reserved by the current connection as well as backup resources denoting sharable slots. However, yellow slot indicates non-sharable slots that it is being reserved by a connection whose primary path shares a link or links with the current connection request's primary path in case its primary path is provisioned.

\section{A. First-Fit}

Search for the available spectrum segment is done in the ascending order of frequency slots starting from Path 1 . As shown in Fig. 1 frequency slots $\mathrm{f} 1$ and $\mathrm{f} 2$ in links $(\mathrm{a}, \mathrm{b})$ and $(\mathrm{b}, \mathrm{c})$ are occupied by other primary paths. For frequency slots $\mathrm{f} 3$ and $\mathrm{f} 4$, the algorithm detects an availability as shown in Fig. 1 (b) along Path 1. Upon this detection, the search is halted without proceeding the remaining slots and paths. Then, primary path for transmission and backup path for reservation are provisioned. First found spectrum slice and the related path are selected as the solution for the primary path. In case there is no so $^{2}$ lution, the connection request is blocked.

\section{B. Minimum Free Spectrum Block Consumption (MFSB)}

In this scheme, primary path routing and spectrum assignment is handled by First-Fit. For the backup resource assignment, among all the candidate paths, the path which

\section{Minimum Free Spectrum Block Consumption (MFSB)}

In this scheme, primary path routing and spectrum assignment is handled by First-Fit. For the backup resource assignment, among all the candidate paths, the path which provides the solution that would lead to the minimum additional number of free frequency slots is chosen. In this manner, authors aim to increase the sharability among backup paths as well as the efficiency of reserved frequency slots utilized by backup paths.

To illustrate, MFSB algorithm examines all the three candidate paths and frequency slots shown in Fig. 1 (b). For Path 1, some assigned slots in the constituting links prevent the new assignment through the links' frequency slots $\mathrm{f1}-\mathrm{f} 2$ and $\mathrm{f5}-\mathrm{f6}$. Frequency slots f3-f4 are available along the path and satisfy the routing and spectrum constraints. However, it consumes five free slots in case it is reserved. In Path 3, there are two such alternatives, f4-f5 and f5-f6. Nevertheless, they both cause three free frequency slots consumption. In Path 2, a backup path composed in f3-f4 slots consumes only a single free slot. Thus, this solution is selected.

\section{Primary First-Fit Modified Backup Last-Fit (PF-MBL)}

We propose a novel algorithm for backup path provisioning based on the idea of separating primary and backup resources on different parts of the spectrum, i.e., different ends of the links' spectrum in order to keep less fragmented space for backup spectrum assignments, thus less fragmented network. Our approach focuses on grouping together the backup resources and not allowing them to mix with primary.

To illustrate, our algorithm examines all the three paths shown in Fig. 1 (b). From this perspective, the right-most solution is tried to be found. For Path 1, some assigned slots in the constituting links prevent the new assignment through the slots $\mathrm{f} 1-\mathrm{f} 2$ and $\mathrm{f5}-\mathrm{f} 6$ as shown in Fig. 1 (b). Frequency slots $\mathrm{f3}-\mathrm{f} 4$ are

\footnotetext{
${ }^{1}$ Derived from the figure in [4]
} 
available along the path and satisfy the routing and spectrum constraints. The right-most slot is f3. Similarly, a backup path through f3-f4 slots along Path 2 is feasible. In Path 3, there are two available sets, $\mathrm{f} 4-\mathrm{f} 5$ and $\mathrm{f} 5-\mathrm{f} 6$ with the right-most choice as f5-f6.

In order to avoid routings through longer paths and higher amount of spectral resource consumption, a hybrid penalty function is applied as shown in Eqn. (1) where $c_{1}$ and $c_{2}$ represents two weight adjustment values between two objectives. $c_{1}$ denotes the weight for prioritizing the assignment of right-most spectrum slots where $c_{2}$ represents the weight for prioritizing the number of frequency slots assigned.

$$
\text { Hybrid Penalty }=\left(W-f_{a}\right) \times c_{1}+\left(f_{z}-f_{a}+1\right) \times c_{2}
$$

$W$ represents the total number of frequency slots on a link which is constant and $\left(f_{z}-f_{a}+1\right)$ gives the required number of slots for corresponding route assignment. $c_{1}$ is a constant smaller than 1 and depends on the topology of the network. $c_{2}$ takes either 0 or 1 . When it takes 0 , formula only depends on the first criterion. In this case, the algorithm selects the solution having the set with the highest numbered starting frequency slot. By adjusting the $c$ values, it is possible to tune the performance of the algorithm.

\section{ILLUSTRATIVE NUMERICAL RESULTS}

NFSNET (14 nodes, 21 links, average node degree=3.14) sample backbone network topology has been taken into account for performance comparison. For the modulation format assignment, values in Table I is considered. It is assumed that each fiber has a contiguous spectral window ( $\mathrm{C}$ band) and a total spectrum of $4000 \mathrm{GHz}$ as 320 frequency slots with the width of $12.5 \mathrm{GHz}$. $\mathrm{k}$ is set to four in k-shortest path algorithm and the guard band is assumed to be one slot. We set the requested bit rates uniformly distributed from $10 \mathrm{~Gb} / \mathrm{s}$ to 400 $\mathrm{Gb} / \mathrm{s}$. Connection requests will be uniformly distributed between all source-destination pairs in the graph. Traffic arrival process will be Poisson with $\lambda$ connection requests per unit time and the traffic holding time will obey negative exponential distribution with a mean value of $\mu .250000$ connections are simulated for each algorithm for five different network loads. Results corresponding to the first 10000 of connections are discarded considering the warm up period before the system reaches a steady-state.

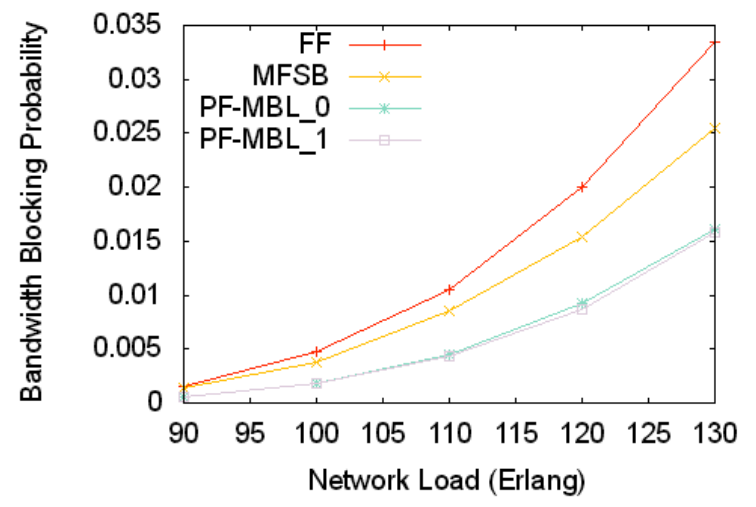

Figure 2. Bandwidth Blocking Probability vs. Network Load (Erlang)
The performance of our proposed algorithm is demonstrated in comparison with two existing solutions FF and MFSB. Additionally, $c_{1}$ in hybrid penalty function of PF-MBL is chosen as $0.34 . c_{2}$ takes both 0 and 1 in order to analyze the tradeoff. Hence, PF-MBL_0 represents the case where $c_{2}$ is 0 . In this case, the formula tries to maximize the starting frequency number among solution sets of the candidate paths. PF-MBL_1 represents the case where the size of the spectrum segment and its position both have influence on choosing the path. In other words, formula tries to minimize the number of frequency slots for the assignment and to maximize the starting frequency number among solution sets of the candidate paths at the same time. Blocking probability and bandwidth blocking probability will be used to quantitatively show the performance of the systems. Shareability, spectrum utilization and fragmentation will be exploited to analyze the performance differences. Performance metrics are explained further in the following subsections.

\section{A. Blocking and Bandwidth Blocking Probability}

Fig. 2 and 3 plot the bandwidth blocking probability (BBP) and the connection blocking probability (BP) ratios of the heuristic algorithms First-Fit, Minimum Free Spectrum Block Consumption (MFSB) and Primary First-Fit Modified Backup Last-Fit (PF-MBL) as the network load increases. PF-MLF_0 and PF-MLF_1 significantly outperforms existing approaches in both figures. PF-MLF_1 achieves savings over FF between $52 \%$ to $65 \%$ and over MFSB savings between $38 \%$ to $59 \%$ in terms of bandwidth blocking probability while its connection request acceptance ratio varies from $99 \%$ to $99,99 \%$. Likewise, PF-MLF_1 outperforms FF by $53 \%$ up to $65 \%$ and similarly achieves savings over MFSB between $38 \%$ to $59 \%$ in terms of blocking probability while its bandwidth acceptance ratio varies from $98,5 \%$ to $99.9 \%$.

\section{B. Sharability and Utilization of Spectral Resources}

Fig. 4 shows the Shareability of the backup resources calculated as the time weighted average of the ratio of total serviced backup resources for each backup path over total assigned backup resources. Note that assigned backup resources will be smaller than serviced resources since backup sharing is enabled. In all algorithms shareability increases when the network load grows, which is rational since the number of connections that will possibly share the frequency slots also increases. It is interesting to observe that, MFSB has the highest sharability

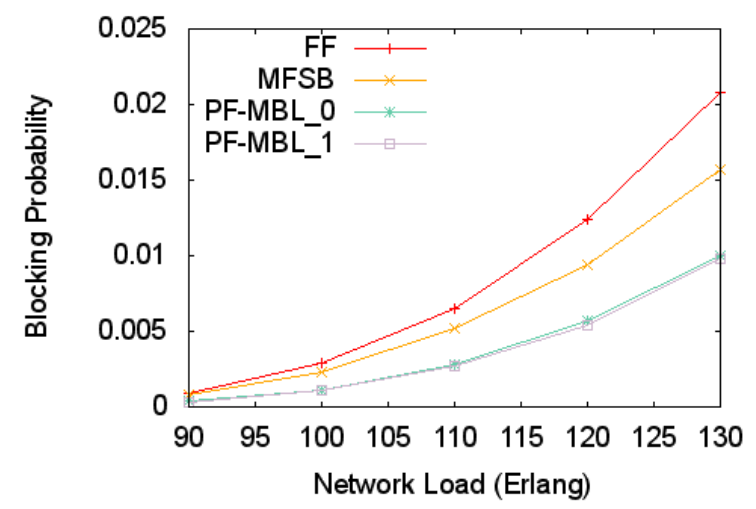

Figure 3. Connection blocking probability vs. Network Load (Erlang) 


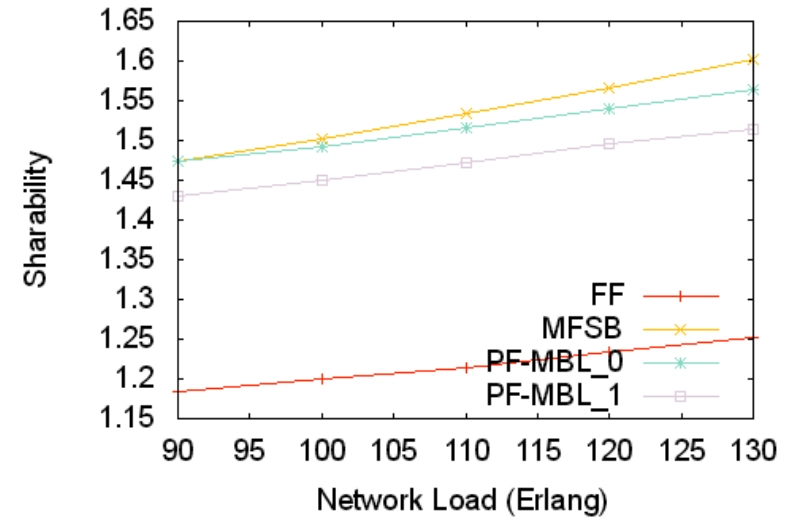

Figure 4. Backup Spectrum Shareability vs. Network Load (Erlang)

closely followed by PF-MBL_ 0 and PF-MBL_ 1 which is also reflected in the utilization of spectral resources shown in Fig. 5. This is because PF-MBL_0 tends to select the longer paths requiring wider spectrum segments in case these segments are the furthest from primary path resource side among competing paths. MFSB surpasses PF-MBL_0 and PF-MBL 1 since it explicitly focuses on increasing the shareability whereas PFMBL implicitly helps shareability increase through separating the resource allocation of primary and backup paths. Nevertheless, PF-MBL_1 algorithm still keep high shareability ratio close to MFSB (only 3 to 5\% drop) and achieves around $20 \%$ higher shareability than FF.

Fig. 5 shows the spectrum utilization ratio for three different approaches. Even though FF rejects the highest number of connection requests, it attains the highest spectrum utilization ratio among the algorithms. This is the result of having the lowest shareability and having highly fragmented links (See Fig. 6.) compared to PF-MBL. MFSB performs the lowest level of spectrum utilization ratio, owing to the keen dedication on efficiently increasing the shareability, closely followed by PF-MBL especially when $c_{l}$ is equal to 1 . However, MFSB's performance comes with a very high cost of BP and BBP compared to PF-MBL. This implies that networks employing MFSB are unable to find primary-backup path pairs satisfying spectrum assignment constraints although there are more available spaces over the network.

\section{Fragmentation}

Fragmentation formula used in [8] and [9] is adopted which also measures hard-disk fragmentation. If all free spectrum slots assemble side by side with no utilized frequency slot in between, it means that there is no fragmentation in a given link. However, if free segments are distributed over the link, fragmentation is proportional to the ratio of the size of the largest free segment over total free frequency slots on a link. Fig. 6 shows the average spectral fragmentation occurred in three different algorithms. FP-MBL significantly outperforms the existing approaches explaining the achieved good performance in terms of BP and BBP. By trading slightly more spectral resources compared to MFSB as shown in Fig. 5, FP-MBL achieves a significant reduction in fragmentation ratio. These results show that separating primary paths and backup paths

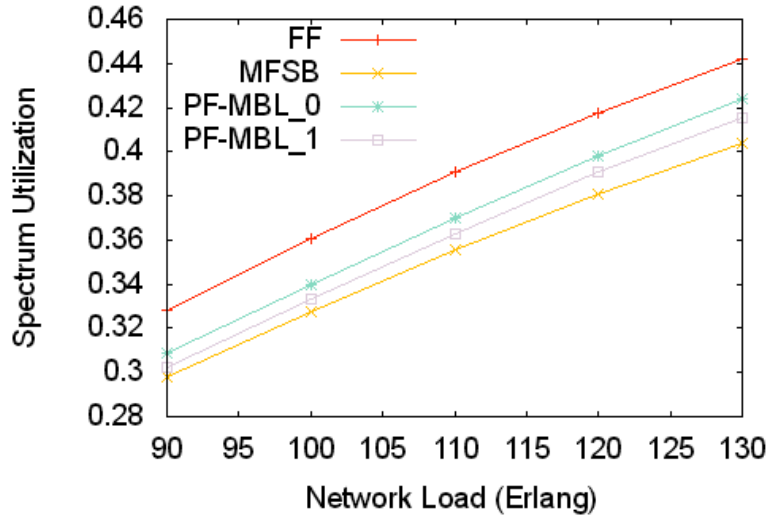

Figure 5. Spectrum Utilization vs. Network Load (Erlang)

helps reducing the spectrum fragmentation by $18 \%$ up to $27 \%$ in the network compared to FF while providing lower BP and BBP than existing approaches. It is interesting to see that MFSB performs the worst in terms of fragmentation, demolishing its good performance in terms of total consumption in spectral resources.

\section{CONCLUSION}

In this paper we have studied shared path protected routing, modulation format and spectrum assignment (SPPRMLR) problem under dynamic traffic conditions. We have discovered that considering solely the total consumption of spectral resources and spectral efficiency do not always lead to good performance in terms of bandwidth blocking probability (BBP). Fragmentation is an important aspect to consider in primary and backup spectrum assignment. Taking this in mind, we have developed a novel SPP-RMLR algorithm that is based on the idea of avoiding to mix primary spectral resources with the backup resources. Moreover we have proposed a penalty function finding a balance between different objectives, total spectral resource usage and BBP.

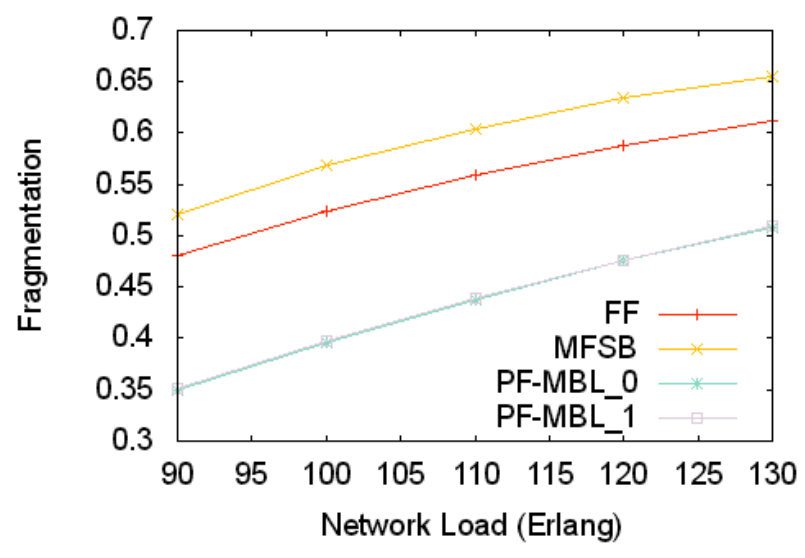

Figure 6. Fragmentation vs. Network Load (Erlang) 


\section{REFERENCES}

[1] A. Saleh and J. M. Simmons, "Technology and architecture to enable the explosive growth of the internet," in IEEE Communications Magazine, vol. 49, no. 1, pp. 126-132, January 2011.

[2] O. Gerstel, M. Jinno, A. Lord, and S. J B Yoo, "Elastic optical networking: a new dawn for the optical layer?," in IEEE Communications Magazine, vol. 50, no. 2, pp. 12-20, February 2012.

[3] X. Shao, Y. Yeo, Z. Xu, X. Cheng, and L. Zhou, "Shared-path protection in OFDM-based optical networks with elastic bandwidth allocation," in Optical Fiber Communication Conference and Ex- position (OFC/NFOEC), 2012

[4] B. Chen, J. Zhang, Y. Zhao, J. Liu, S. Huang, W. Gu, and J. P. Jue, "Minimum spectrum block consumption for shared-path protection with joint failure probability in flexible bandwidth optical networks," in Optical Fiber Communication Conference and Exposition (OFC/NFOEC), 2013.
[5] A. Bocoi, M. Schuster, F. Rambach, M. Kiese, C. A. Bunge, and B. Spinnler, "Reach-dependent capacity in optical networks enabled by OFDM," in Optical Fiber Communication Conference and Exposition (OFC/NFOEC), 2009.

[6] K. Christodoulopoulos, I. Tomkos, and E.A. Varvarigos, "Elastic bandwidth allocation in flexible OFDM-based optical networks," Journal of Lightwave Technology, vol. 29, no. 9, pp. 1354-1366, May 2011.

[7] J. Y. Yen, "Finding the K shortest loopless paths in a network," Management Science, vol. 17, no. 11, pp. 712-716, 1971.

[8] H.G. Baker, "Memory Management," in proceedings International Workshop IWMM 95, Kinross, UK, September 29 - 29, 1995. Lecture Notes in Computer Science. Springer, vol. 986, 1995.

[9] A. Rosa, C. Cavdar, S. Carvalho, J. Costa, and L. Wosinska, "Spectrum allocation policy modeling for elastic optical networks," in High Capacity Optical Networks and Enabling Technologies (HONET), 2012, 9th International Conference on, pages 242-246, Nov. 2012. 